\title{
LA TEORÍA DE LA DEGENERACIÓN Y LA PROFESIONALIZACIÓN DE LA PSIQUIATRÍA EN ESPAÑA (1876-1920)*
}

\author{
Ricardo Campos Marín \\ Investigador contratado - Depto. de Historia de la Ciencia. - CEH - CSIC \\ Duque de Medinaceli, 6. 28014 Madrid (España)
}

\section{RESUMEN}

El presente trabajo aborda el estudio de la difusión del degeneracionismo en España en relación con las estrategias de profesionalización de la psiquiatría. En este sentido, se analiza como entre 1876-1900 el degeneracionismo será utilizado profusamente ante los tribunales, mientras que en la práctica clínica, los psiquiatras se mostrarán mas reticentes. Esta situación cambiará sustancialmente desde 1900, al variar las condiciones profesionales, políticas y sociales del país. Desde comienzos del siglo XX, el degeneracionismo tendrá una mayor difusión entre los alienistas españoles, desapareciendo en su utilización la separación entre el ámbito clínico y el jurídico.

\section{SUMMARY}

This paper approaches to the study of the diffusion of degeneracionism in Spain related to the strategies of professionalization of psychiatry. In this way, we analize how degeneracionism was profusely tanken to court from 1876 to 1900 , while psychiatrists were more deceptive in their clinical practice. Since 1900, this situation will substantially be modified because of changes in political, professional and social conditions in the country. Since the begining of the $20^{\text {th }}$ Century, the degeneracionism will have got a greaten diffusion among, Spanish alienists wearing off its division betwen clinical or juridical fields.

\section{INTRODUCCIÓN}

Hasta la fecha los escasos estudios que han abordado la introducción de la teoría de la degeneración en España se han ocupado de analizarla en relación con comportamientos antisociales de gran repercusión como el alcoholismo y la criminalidad, sin abordar el estudio pormenorizado de su recepción en el ámbito estrictamente psi-

* Trabajo realizado en el marco del proyecto de investigación PB94-0064 de la DGYCIT. 
quiátrico $^{1}$. Poco se ha escrito sobre esta compleja cuestión en la que confluyeron diversos aspectos de la realidad psiquiátrica y social, que modelaron el proceso de aceptación y reelaboración de la teoría de la degeneración por parte de los frenópatas españoles ${ }^{2}$.

En un interesante trabajo, I. Dowbiggin, demuestra cómo la psiquiatría francesa de la segunda mitad del siglo XIX se acogió paulatinamente a las interpretaciones hereditarias de la enfermedad mental, en especial a la degeneracionista, como estrategia de profesionalización y legitimación de su saber científico ante el Estado y la opinión pública ${ }^{3}$. Hacia 1860, según nos cuenta el autor citado, el alienismo francés se encontraba en una situación de «estado de sitio» por parte de la opinión pública que denunciaba, como crítica al sistema político erigido por Napoleón III, el fracaso del manicomio como institución terapeútica y los constantes abusos que se cometían tanto en los procesos de ingreso como en el interior de estas instituciones. Las denuncias ponían de relieve la contradicción entre los postulados del tratamiento moral y la verdadera situación de los manicomios, donde se hacía imposible llevarlo a cabo.

Las implicaciones científicas y profesionales de esta situación eran evidentes. Durante años los alienistas franceses habían intentado poner de relieve la naturaleza física de la locura para presentar el alienismo como una ciencia específica y diferenciada de las demás. El problema al que se enfrentaban era localizar la causa física de la locura, dado que los estados psicológicos eran el objetivo primordial de la clínica psiquiátrica. Sin una explicación organicista de estos estados, el alienismo estaba amenazado por la intervención de otros grupos que consideraban la locura como una cuestión meramente espiritual o moral. Este problema se veía agravado por la realidad de la práctica psiquiátrica, basada en el tratamiento moral ${ }^{4}$. I. Dowbiggin señala que tras el fracaso de la Anatomía Patologica y la frenología para explicar la natura-

\footnotetext{
1 Pueden verse al respecto, CAMPOS MARín, R; HUeRTAS, R. (1992), «Alcoholismo y degeneración en la medicina positivista española», Revista de la Asociación Española de Neuropsiquiatría, 12, pp. 125-129; CAMPOS MARín, R. (1995a), «La recepción de la teoría de la degeneración francesa en el discurso antialcohólico español (1890-1915)», en ARQUIOLA, E. y MARTíNEZ, J. (Coords.). Ciencia en expansión. Estudios sobre la difusión de las ideas científicas y médicas en España (Siglos XVIII-XX), Madrid, Editorial Complutense, pp. 449-467; CAMPOS MARÍn, R. (1997). Alcoholismo, Medicina y Sociedad en España. (1876-1923), Madrid, CSIC; HUERTAS, R; MARTíNEZ PÉREZ, J. (1993), «Disease and crime in the spanish positivist psychatry», History of Psychiatry, 4, pp. 459-481.

2 Véase a este respecto HUERTAS, R. (1995a), «Sobre la recepción del degeneracionismo psiquiátrico en España: la obra de Mateo Bonafonte», en: ARQUIOLA, E. y MARTínEZ, J. (Coords.), Ciencia en expansión. Estudios sobre la difusión de las ideas científicas y médicas en España (Siglos XVIII-XX), Madrid, Editorial Complutense, pp. 521-534. Proximamente podrá verse también CAMPOS MARÍN, R. (1999) «La teoría de la degeneración y la clínica psiquiátrica en España. (1876-1920), Dynamis, vol. 19 (en prensa)

3 DowbIGGIN, I. (1993). La folie héréditaire ou comment la psychiatrie française c'est constituée en un corps de savoir et de pouvoir dans la seconde moitié du XIXeme siècle, Paris, Epel.

4 Ibid, pp. 127-153. Sobre esta cuestión también puede verse, HUERTAS, R. (1992). Del manicomio a la salud mental. Para una historia de la psiquiatría pública, Madrid, FISS, pp. 43-88.
} 


\section{LA TEORÍA DE LA DEGENERACÓN Y LA PROFESIONALIZACIÓN DE LA PSIQUIATRÍA}

leza somática de la enfermedad mental, los psiquiatras franceses se vieron obligados a reaccionar para adoptar un modelo clínico de patología mental que convenciera al Estado, a los médicos y a la opinión pública de que eran los poseedores de una ciencia especial, capaz de probar la naturaleza biológica de la locura. La teoría de la degeneración fue el modelo ideal que resolvía estos problemas, al conectar, sobre todo desde la derrota de 1870 y de la Comuna de París, con las inquietudes y ansiedades de la sociedad francesa. En este sentido, el degeneracionismo era un poderoso instrumento de profesionalización y de interpretación de la realidad social y política francesa, que facilitaba el deseado monopolio asistencial de la locura 5 .

Por su parte los frenópatas españoles fueron bastante más modestos en sus objetivos y lectura de la teoría de la degeneración.

En líneas generales, durante el periodo 1876-1900, la actitud de los alienistas fue de rechazo o indiferencia clínica hacia el degeneracionismo, como teoría de la interpretación de la naturaleza de la locura. Sólo a partir de mediados de la década de 1890 se produjo una mayor proliferación de los trabajos degeneracionistas, pero sus inquietudes no revistieron un especial interés por las interpretaciones sociales y colectivas. Su atención se centró principalmente en cuestiones estrictamente clínicas como el papel de la herencia, los estigmas físicos y psíquicos del individuo degenerado y por los problemas de clasificación de la locura que entrañaba el degeneracionismo, pero siempre circunscritos al individuo degenerado y a su familia, sin que existiera una visión apocalíptica de la degeneración de la raza, ni una especial insistencia en equiparar la degeneración a la decadencia de la sociedad. Su discurso se centraba exclusivamente en el diagnóstico clínico sin que se introdujeran elementos sociólogicos susceptibles de convertir la teoría de la degeneración en un marco interpretativo de la sociedad española finisecular o en un instrumento de acción social ligado a la higiene social. Este papel correspondió a los higienistas y a los médicos sociales, mucho más vinculados a los problemas sociales y económicos que acuciaban al país ${ }^{6}$. Sólo desde principios de siglo, cuando los alienistas vayan abriendo su

5 DowBIGGIN (1993), pp. 155-213. En los últimos años varios estudios han remarcado en relación con la profesionalización de las especializades médicas, la capacidad de oferta, por parte de un colectivo profesional interesado, de una mercancia claramente distinguible sobre la que poder reinvidicar un monopolio. Sirvan como botón de muestra: HoFOSs, D. (1986), «Health Professionals: The Origins of Species», Social Science and Medecine, 22, pp. 201-209; HALPERN, S.A. (1988), American Pediatrics. The Social Dynamics of Professionalism, 1880-1980, Berkeley, University of California Prees; MEDINA, R. (1993), Estrategias profesionalizadoras en el origen de una especialidad médica. El caso de la radioterapia española. 1885-1936, Tesis doctoral. Universidad de Granada. Microfichas; HUERTAS, R. (1995b), «La psiquiatría española del siglo XIX. Primeros intentos de institucionalización», en I Congreso de la Sociedad de Historia y Filosofía de la Psiquiatría. Un siglo de psiquiatría en España, Madrid, Extra editorial, pp. 21-40.

6 Sobre este aspecto podrá verse CAMPOS MARín, R. (1998), «La teoría de la degeneración y la medicina social en España en el cambio de siglo», Llull, vol. 21, n 41, pp. 333-356. 
campo de acción a la sociedad y comiencen a reinterpretar el papel del manicomio como institución terapéutica, aparecerán trabajos con mayores implicaciones sociales ${ }^{7}$.

Sin embargo, durante ese mismo período (1876-1900), las posiciones de los alienistas fueron bien distintas cuando actuaban como peritos ante los tribunales. En este ámbito, el degeneracionismo fue utilizado a discreción para demostrar la patología mental del procesado y pronunciarse en favor de su internamiento en un manicomio. Desde este punto de vista, las implicaciones sociales del degeneracionismo fueron mayores pues los alienistas elaboraron una doctrina de la defensa social que tuvo amplia repercusión en la sociedad finisecular.

Esta doble actitud frente a la teoría de la degeneración respondía, a nuestro juicio, a las estrategias de profesionalización e institucionalización de la psiquiatría en sus diferentes campos de acción.

Aspectos clínicos y científicos, de profesionalización e institucionalización de la disciplina y de organización de la asistencia psiquiátrica estuvieron presentes y marcaron su impronta en todo este proceso. En este trabajo pretendemos analizar la introducción, aceptación y difusión del degeneracionismo entre los psiquiatras españoles relacionándolo con las estrategias de profesionalización e institucionalización de la psiquiatría durante la España de la Restauración. En este sentido, partimos de la hipótesis de que la teoría de la degeneración en España en el último cuarto del siglo XIX, no fue aceptada como corpus teorico completo, sino que fue instrumentalizada por los alienistas según el contexto en que desempeñaran su actividad profesional. Concretamente, intentaremos demostrar que el degeneracionismo tuvo una diferente acogida, dependiendo de si los psiquiatras pretendían legitimarse en el campo clínico y asistencial o en terrenos de mayor repercusión pública de su actividad como los grandes procesos judiciales.

Para ello, analizaremos una serie de escritos teóricos y prácticos de los alienistas españoles del periodo, que ponen de relieve esta ambigüedad ante la teoría de la degeneración.

\section{LA PRUDENCIA CLÍNICA ANTE EL DEGENERACIONSIMO}

En 1876, diecinueve años después de que Morel formulase su teoría de la degeneración ${ }^{8}$, J. Giné y Partagás publicaba su Tratado Teórico-Práctico de Frenopatolo-

\footnotetext{
7 CAMPOS MARín, R. (1995b), «Psiquiatría e higiene social en la España de la Restauración», en $I$ Congreso de la Sociedad de Historia y Filosofía de la Psiquiatría. Un siglo de psiquiatría en España, Madrid, Extra Editorial, pp. 53-63; CAMPOS MARín, R. (1995c), «Higiene mental y Reforma de la asistencia psiquiátrica en España (1900-1931)», Documentos de Psiquiatría Comunitaria, 7, pp. 199-217.

8 MOREL, B.A. (1857), Traité des dégénérescences physiques, intellectuels et morales de l'espèce humaine et des causes qui produisent ces variétés maladives, París, B. Baillière.
} 


\section{LA TEORÍA DE LA DEGENERACÓN Y LA PROFESIONALIZACIÓN DE LA PSIQUIATRÍA}

gía, en el que abordaba desde un enfoque clínico los diversos aspectos relacionados con la vesania. ${ }^{9}$ Giné reivindicaba en su obra la autonomía de la «frenopatología» como rama específica de la medicina. Sólo el frenópata con el bagaje de sus estudios de medicina estaba, a su juicio, autorizado para estudiar la enajenación mental y establecer la terapia adecuada. Por lo que se refiere a la concepción de la enfermedad mental, Giné se alineaba con la corriente positivista y organicista al considerar que ésta no era una afección o un estado anómalo del espíritu sino «una manifestación patológica del cerebro» ${ }^{10}$.

Sin embargo, a pesar de su adscripción al somaticismo, Giné no aceptaba la teoría moreliana de la degeneración. En el capítulo XVI, dedicado a la clasificación de las enfermedades mentales, escribía que:

\footnotetext{
«el objeto fundamental de una buena clasificación clínica ha de ser proporcionar el mayor número posible de utilidades prácticas, de modo que, al determinar junto al enfermo que su afección pertenece a este o al otro grupo nosológico, nazcan en la mente, los conceptos más útiles desde el punto de vista de la etiología, de la semeiotica y de la terapeútica» ${ }^{11}$.
}

La «utilidad clínica», según Giné, habría de ser el criterio que debía regir las clasificaciones de la locura. Desde este enfoque estrictamente clínico propone la utilización de «cuanto hay de útil en los diferentes ensayos de clasificación de las enfermedades mentales», en tanto y cuanto sirvan al alienista de pauta en la clínica, renunciando, a:

\footnotetext{
«todo conato de clasificación basada en el sitio de las lesiones que producen transtornos funcionales, porque, hoy por hoy, esta idea sería más especulativa que práctica en razón a que no tenemos suficientes datos para establecer estas localizaciones, y aun cuando los tuvieramos, ni sabríamos ponderar con precisión el valor clínico de estas lesiones, ni tendríamos a nuestra mano una terapéutica pronta a obrar según el sentido de las indicaciones sugeridas por el sitio y naturaleza del daño encefálico» ${ }^{12}$.
}

Tras esta declaración, que resaltaba la necesidad de una clasificación apropiada para la práctica clínica, Giné rechazaba la teoría de la degeneración de Morel, «como guía de diagnóstico», argumentando que:

«No tratándose de causas que tengan siempre un mismo modo de obrar ni conduzcan constantemente a unos mismos resultados, la clasificación de Morel es de todo punto inad-

9 GINÉ y PARTAGÁS, Juan (1876). Tratado Teórico-Práctico de Frenopatología o Estudio de las Enfermedades Mentales, Madrid, Moya y Plaza.
10 GiNÉ y PARTAGÁs (1876), p. 45
11 Ibid, p. 238.
12 Ibid, p. 239. 


\section{RICARDO CAMPOS MARÍN}

misible, pues por ella se asimilan estados frenopáticos muy diversos y resultan disociados otros que presentan notables analogías» 13 .

Por tanto, al hilo de sus palabras, su oposición al degeneracionismo parece obedecer exclusivamente a criterios clínicos, aunque como intentaremos demostrar más adelante, éstos estuvieron ligados a estrategias de profesionalización.

Por su parte, A. Galcerán y Granés, discípulo de Giné y uno de los alienistas más representativos de finales del siglo XIX y primeros años del XX, tampoco parece haber prestado especial atención al degeneracionismo durante los años a que nos referimos. Sus artículos en la Revista Frenopática Barcelonesa, publicados en la década de 1880, no abordan la cuestión y en su libro Neuropatología y Psiquiatría general de $1895^{14}$, mera recopilación de sus lecciones impartidas en la universidad, no menciona el degeneracionismo ${ }^{15}$. Más adelante, a partir de 1900 , como veremos, sí prestó más atención a la degeneración, incluyéndola en sus trabajos clasificatorios de la enfermedad mental.

El caso de J.M. ${ }^{a}$ Esquerdo, director y propietario del Manicomio de Carabanchel, es algo más complejo. Esquerdo, a diferencia de sus colegas catalanes, fue poco prolífico en escritos clínicos y la mayor parte de su escasa obra tuvo un carácter publicístico y estuvo dirigida a reclamar el papel de los alienistas como expertos ante los tribunales. Es muy posible, aunque lo desconocemos, que en la práctica clínica en su manicomio y como consecuencia de su optimismo terapeútico y de la extracción social de su clientela, Esquerdo no diagnosticara normalmente casos de degeneración. J. Vera, uno de sus principales discípulos, escribió a principios de la década de 1880 dos trabajos clínicos en los que el degeneracionismo estaba ausente ${ }^{16}$. Sin embargo, Esquerdo, en uno de sus pocos trabajos clínicos publicado en la Revista Clínica de los Hospitales entre 1889 y 1890 , relata un caso de excitación maniaca en el que la paciente tuvo una prole marcada «por el estigma de la degeneración vesánica» ${ }^{17}$. Es posible que la fecha de publicación del artículo tenga que ver con su men-

13 Ibid, p. 240.

14 GAlCERAN y GRANÉS, A. (1895), Neuropatología y Psiquiatría General, Barcelona, Imp. C.P. Caridad.

15 Sobre la obra de Galcerán véase: DOMENECH, E y CORBELLA, J. (1969), «La obra psiquiátrica de Arturo Galcerán Granés», Asclepio, 21, pp. 157-171; REY GoNZÁlEZ, A. M. (1985), «Clásicos de la psiquiatría española del siglo XIX: Arturo Galcerán Granés (1850-1919)», Revista de la Asociación Española de Neuropsiquiatria, n. ${ }^{\circ}$ 13, Abril/Junio, pp. 223-233.

16 VERA y LOPEZ. J. (1880), Estudio clínico de la parálisis general de los enajenados, Madrid, Moya y Plaza Editores; VERA Y LOPEZ, J. (1882) La función de los conductos semicirculares. Estudio experimental, Madrid, Establecimiento Tipográfico de E. Cuesta.

17 ESQUERDO, J. M. (1889), «De la locura histérica», Revista Clínica de los Hospitales, Tomo I, p. 339; Algunos autores señalan que en 1890 Esquerdo publicó un Tratado de Frenopatía que no hemos 


\section{LA TEORÍA DE LA DEGENERACÓN Y LA PROFESIONALIZACIÓN DE LA PSIQUIATRÍA}

ción expresa del degeneracionismo. Hay que tener en cuenta que en 1890, varios alienistas de su entorno habían participado en importantes procesos judiciales en los que ponían de relieve la degeneración del procesado y que en 1888 el propio Esquerdo había escrito el prólogo a la obra de V. Garrido y Escuin La cárcel o el manicomio en la que éste último utilizaba el degeneracionismo como hilo conductor. Con todo, resulta díficil concluir si J. M. ${ }^{a}$ Esquerdo durante la década de 1880 utilizó con regularidad la teoría de la degeneración en su práctica clínica.

\section{EL DEGENERACIONISMO ANTE LOS TRIBUNALES.}

Lo que parece claro es que la ambigüedad, rechazo o indiferencia clínica hacia el degeneracionismo varió sustancialmente en el ámbito de los tribunales.

En la década de 1880, los alienistas formados por Esquerdo o con importantes conexiones con él se mostraron decididamente degeneracionistas en sus actuaciones ante los tribunales de justicia. El propio Esquerdo en su célebre conferencia «Locos que no lo parecen», al exponer su diagnóstico sobre la enfermedad mental de Garayo, acusado de seis asesinatos con violación, estaba cercano al degeneracionismo aunque no mencionase el término ni a sus mentores, y utilizara todavía el concepto de monomanía para justificar su diagnóstico. En cualquier caso, buena parte de los razonamientos de Esquerdo se apoyaban en la descripción de los estigmas físicos de Garayo, en su comportamiento psíquico y moral y en sus antecedentes hereditarios para demostrar su enfermedad mental y la necesidad de recluirlo en un manicomio ${ }^{18}$.

Más claro en este sentido fue el proceso al cura Galeote acusado de asesinar al obispo de Madrid-Alcalá. El proceso que transcurrió entre septiembre y octubre de 1886 contó con los informes de tres jóvenes alienistas que habían seguido el magisterio de Esquerdo: L. Simarro, J.M. ${ }^{a}$ Escuder y J. Vera. Si bien este último hizo un informe estrictamente clínico, sin entrar en consideraciones antropológicas para determinar la locura de Galeote, tanto Simarro como Escuder recurrieron a la descripción de los estigmas físicos, psíquicos y a la herencia para justificar su reclusión en el manicomio. Ambos, a partir de estos signos, calificaban a Galeote como «degenera-

conseguido localizar. La referencia la hemos encontrado en LLORCA BAUS, C. (1984), José María Esquerdo: el gran desconocido, Alicante, Ayuntamiento de Villajoyosa.

18 ESQUERDO, J. M. a (1881), Locos que no lo parecen. Garayo el Sacamantecas, Madrid, Imprenta y Estereotipia de El Liberal; Unos años antes Esquerdo se mostraba mucho más cercano al paradigma de la monomanía para justificar la intervención de los psiquiatras ante los tribunales y diagnosticar la locura de los encausados. Véase a este respecto: ESQUERDO, J. M. ${ }^{a}$ (1878), Preocupaciones reinantes acerca de la locura. Conferencia dada en el Ateneo el día 5 de diciembre de 1878, Madrid, Imprenta y Estereotipia de Álvarez Hermanos. 
do». ${ }^{19}$ Simarro, que siguió en París entre 1880 y 1885 las enseñanzas de Charcot, Richet y Magnan, ${ }^{20}$ realizó una descripción física de Galeote en la que confluían una serie de estigmas físicos, que consideraba claros signos de degeneración. También realizó una incursión en los antecedentes hereditarios de Galeote para confirmar su diagnóstico y remarcar que «la importancia que atribuimos a los datos hereditarios no es meramente una opinión personal; está fundada en doctrinas clásicas generalmente aceptadas entre todos los alienistas de Europa,» añadiendo a continuación:

\footnotetext{
«la locura hereditaria tiene tanta importancia, que se ha encontrado una clase entera de individuos en la sociedad que heredan degeneraciones físicas y morales por la enfermedad mental de sus ascendientes, y estos individuos forman una escala de degeneración» ${ }^{21}$.
}

Si bien no nombra a ninguno de los especialistas de los que es deudor, es evidente que se refiere a los maestros de la teoría de la degeneración y la escuela antropológica italiana.

A partir de estos datos dictaminaba la irresponsabilidad de Galeote y le definía como un degenerado:

«Hay varios grados de locura: el idiotismo, o estado deficiente de desarrollo moral, que se manifiesta desde el nacimiento del individuo; la imbecilidad, o deficiencia del desarrollo moral después del nacimiento, la debilidad degenerativa y el estado de pura anomalía intelectual. De estos cuatro grados, nosotros creemos que puede colocarse la locura de Galeote en el caso tercero, o sea la debilidad degenerativa»22.

Llama la atención al leer el texto de Simarro la linealidad de sus argumentos para declarar a Galeote degenerado. Sin duda, esta actitud respondía a la necesidad de convencer a unos magistrados que desconfiaban de los alienistas y del papel que pretendían arrogarse.

19 Los informes de Vera, Simarro y Escuder se encuentran reproducidos en VARELA, J y ALVAREZURIA, F. (1979), El cura Galeote asesino del Obispo de Madrid-Alcalá, Madrid, La Piqueta, pp.99-108. También puede consultarse sobre este asunto GALERA, A. (1991), Ciencia y Delincuencia. El determinismo antropológico en la España del siglo XIX, Sevilla, CSIC, pp. 21-25.

20 PUIG-SAMPER, M.A. (1987), «Luis Simarro y las ciencias neurobiológicas», Rev. Asoc. Esp. Neuropsiq, 7, pp. 649-661; CARPINTERO H. (1994), Historia de la Psicología en España, Salamanca, Eudema, pp. 140-141.

21 En VAREla y AlVAREZ-URía (1979), p. 100.

22 Ibidem. 


\title{
LA TEORÍA DE LA DEGENERACÓN Y LA PROFESIONALIZACIÓN DE LA PSIQUIATRÍA
}

Por su parte, J.M. ${ }^{a}$ Escuder llevó a cabo un minucioso estudio psíco-somático de Galeote en el que se daban cita todos los tópicos sobre la cuestión ${ }^{23}$. El sacerdote era un compendio de taras degenerativas que Escuder achacaba a la herencia biológica familiar recibida, que él personalmente se había tomado la molestia de estudiar desplazándose a Vélez-Málaga, localidad natal de Galeote. La conclusión de sus pesquisas no podía ser más contundente:

\footnotetext{
«Cuatro generaciones de la familia de D. Cayetano Galeote y Cotilla han desfilado ante mis ojos, y aún hasta la quinta he podido estudiar, quedando maravillado al contemplar la inmutable ley de la herencia, faltalmente cumplida en ciento sesenta y tres parientes del procesado, de los cuales han muerto noventa y siete y quedan vivos sesenta y seis» 24 .
}

Afirmación cuanto menos chocante por la amplitud del estudio generacional que se atribuye, por la enorme cantidad de fallecidos que parece haber estudiado personalmente y por la contudencia de las leyes hereditarias que no parecen haber perdonado a uno sólo de los 163 parientes de Galeote.

Este «ejemplar» trabajo empírico le llevaba a concluir que en la familia del acusado se cumplía una de las leyes inexorables de los degenerados cual es la tendencia a unirse:

\begin{abstract}
«Cotillas y Galeote han aportado cada uno al fondo común los gérmenes constitutivos de sus diátesis neuropáticas, y juntándose como dos ríos en un solo lecho, al mezclar sus sangres, han transmitido a sus descendientes, difundida en sus venas, la herencia nerviosa que les distingue; cúmplese en ellos la ley de la afinidad patológica que fuerza a buscarse a los individuos neuropáticos, sin duda porque cada ser busca su semejante» 25 .
\end{abstract}

Ambos informes tenían una clara afinidad con el degeneracionismo, y la influencia de Lombroso es patente en la caracterización de un tipo antropológico específico del criminal, así como en las menciones de Escuder al «salto atávico» de algunos miembros de la familia de Galeote.

Otro ejemplo significativo de la actitud receptiva al degeneracionismo lo encontramos en la obra de V. Garrido Escuín, La cárcel o el manicomio, publicada en 1888 y alabada en el prólogo por Esquerdo. ${ }^{26} \mathrm{La}$ obra de Garrido, en sintonía con su maestro, tenía como objetivo reinvidicar el papel del alienista ante los tribunales como

23 Escuder dedicó buena parte de su labor como escritor a relacionar el crimen con la locura, utilizando el degeneracionismo como hilo conductor. Sus dos obras principales son: ESCUDER J.M . Quemas y crímenes, Valencia; ESCUDER, J.M. ${ }^{a}$ (1895), Locos y anómalos, Madrid, Tip. Sucesores de Rivadeneyra.

24 En Varela y AlvareZ-Uria (1979), p. 106.

25 Ibidem.

26 GARRIDO, V. (1888), La Cárcel o el Manicomio. Estudio Médico Legal sobre la locura, Madrid, Administración Casa Editorial de don José María Faquineto. 


\section{RICARDO CAMPOS MARÍN}

el único especialista capacitado para desentrañar las relaciones entre la locura y la criminalidad. Sin embargo, su interés reside en la utilización del degeneracionismo como hilo argumental y principal apoyo científico para justificar su objetivo. A lo largo del libro Garrido va desgranando los principales temas de la teoría de la degeneración, demostrando conocer las principales obras de Morel, Magnan y de Lombroso.

En 1894, se publicó el dictamen Médico Legal del proceso a Samuel Willie, firmado por quince peritos entre los que destacan las firmas de J. Giné i Partagás, A. Rodríguez Morini y A. Galcerán ${ }^{27}$. El texto que relata el crimen cometido por Samuel Willie, hombre de negocios inglés, acusado de disparar contra sus socios catalanes, ofrece una extensa y pormenorizada historia familiar, personal y clínica del encausado.

Los firmantes del dictamen, tras trazar la genealogía de Willie, concluyen por señalar que en su familia había una importante herencia vesánica. Su bisabuelo paterno y su abuelo materno se suicidaron. El abuelo paterno era alcohólico crónico (ebrioso habitual) y murió loco poco antes del nacimiento de Samuel. Por el contrario sus padres, a juicio de los peritos, no presentaban «sello alguno frenopático, como no se tome por tal su gran religiosidad y su extremada devoción» El panorama sin embargo, cambiaba al referirse a sus hermanos. De los cuatro que tenía, uno «afectado de gran deformidad craneal, murió de coqueluche, a la edad de seis meses, habiendo opinado uno de los profesores que fueron llamados en consulta, que la conformación de su cráneo no prometía sino imbecilidad o idiotismo», mientras que otro presentaba «una asimetría muy acentuada en su semblante por parálisis del nervio facial derecho» cuyo origen no es «periférico, sino nuclear encefálico» y data «de los albores de la segunda infancia» quedando «como reliquia indeleble de un afecto agudo, seguramente hemorrágico, que en aquel tiempo vino a perturbar gravemente la salud de este individuo» ${ }^{28}$. A partir de estos datos, que confirmaban la carga hereditaria en S. Willie, y del análisis de la evolución de su personalidad caracterizada por su «exagerada emotividad y de una grande hiperestesia del sentido moral» concluían por calificarle unánimentente como «degenerado, hereditario y desequilibrado» ${ }^{29}$.

\section{LAS CAUSAS DE LA AMBIGÜEDAD PSIQUIÁTRICA FRENTE A LA TEORÍA DE LA DEGENERACIÓN.}

A la luz de estos datos, parece que entre los años setenta y noventa del siglo XIX, el degeneracionismo tuvo mayor incidencia en el ámbito jurídico que en el clínico.

27 Giné y PaRTAGÁs, J; RodrigueZ-Morini, A. R. y otros (1894), Dictamen Médico-Legal que en el proceso Samuel Willie unanimemente han emitido los peritos nombrados por el Ministerio Fiscal, la acusación privada y la defensa, Barcelona, Imprenta de la Casa Provincial de Caridad.

28 Ibid, pp. 17-18.

29 Ibid, p. 29. 


\section{LA TEORÍA DE LA DEGENERACÓN Y LA PROFESIONALIZACIÓN DE LA PSIQUIATRÍA}

El porqué de esta doble actitud hay que buscarlo, a nuestro juicio, en varios factores de diferente origen pero convergentes y no excluyentes entre sí.

En primer lugar, hay que partir de los aspectos clínicos del problema. No se puede ignorar que durante el periodo estudiado, las influencias clínicas de los alienistas españoles no estuvieron marcadas por el degeneracionismo. Aunque Morel formulara su teoría en 1857, el alienismo español se mantuvo durante mucho tiempo influido por la monomanía para explicar la naturaleza de la enfermedad mental. P. Mata se mostraba firme partidario de la doctrina esquiroliana y es bien conocida su labor en la reforma del código penal de 1870 que se inspira en buena medida en la misma ${ }^{30}$.

Por lo que se refiere al rechazo de J. Giné a la teoría de Morel, hay que tener en cuenta que el alienista catalán es deudor intelectual de J. Guislain cuya clasificación de la locura considera seductora y muy útil desde el punto de vista clínico:

\footnotetext{
«Su idea de hacer derivar la noción del estado frenopático de las seis órdenes de manifestaciones fisiológicas de las facultades psíquicas que sirven de tipo de comparación son muy útiles» ${ }^{31}$.
}

J. Guislain, en sus Lecciones Orales sobre las Frenopatías publicadas originalmente en $1852^{32}$ y traducidas al castellano en 1881 , indica que ante la variedad de expresiones de la enfermedad mental conviene simplificar y «encontrar en este número prodigioso de manifestaciones, tipos elementales, expresiones fundamentales» ${ }^{33}$. Esta expresiones las reduce a seis: Melancolía, Extasis, Manía, Locura, Delirio y Demencia, admitiendo que puedan presentarse en «estado simple» o «compuesto» ${ }^{34}$. El criterio clínico también es preponderante en la obra de Guislain, quien apunta que «la mejor clasificación será siempre la que hable más pronto y de la manera más lúcida a la inteligencia del práctico. En los cuadros nosográficos debe de-

30 Véase ÁlvareZ-URIA, F. (1983), Miserables y locos. Medicina Mental y orden social en la España del siglo XIX, Madrid, Tusquets editores; MARTíNEZ PÉREZ, J. (1995a), «Problemas científicos y socioculturales en la difusión de una doctrina psiquiátrica: la recepción del concepto de monomanía en España», en ARQUIOLA, E y MARTÍNEZ PÉREZ, J. (Coords), Ciencia en expansión. Estudios sobre la difusión de las ideas científicas y médicas en España (siglos XVIII-XX), Madrid, Editorial Complutense, pp. 489-520.

31 GINÉ (1876), p. 241.

32 GuISLAIN, J. (1852), Leçons orales sur les phrénopathies, Bruxelles.

33 GuISLAIN, J. (1881), Lecciones orales sobre las Frenopatías o Tratado Teórico-práctico de las enfermedades mentales, Madrid, Imprenta de Enrique Teodoro, p. 72. En buena medida Guislain estaba anticipando el concepto de psicosis única que posteriormente acuñaría Griesinger y que tanto éxito alcanzó de la mano de la nosología kraepeliana. Sobre la influencia de Guislain en Giné, y sobre la clasificación de la locura en la obra de este último véase: DiÉGUEZ GóMEZ, A. (1998), «El problema de la nosografía en la obra de Giné y Partagás», Asclepio, L (1), pp. 197-219.

34 Guislain (1881), pp. 72-73. 


\section{RICARDO CAMPOS MARÍN}

signarse el fenómeno más saliente y no la esencia íntima, a menos que ésta se presente en estado de evidencia completa.» De ahí que el alienista belga descalifique a Morel en los siguientes términos: «Lo propio diremos de la agrupación etiológica. Morel ha hecho un ensayo brillante en Francia; pero a pesar de todo el prestigio de su envidiable talento, no ha podido hacerle entrar en la ciencia ${ }^{35}$.

Por su parte, J.M. ${ }^{a}$ Esquerdo también se mostró admirador de la obra del alienista belga, aunque mantuvo un distanciamiento crítico, al considerar que su definición de la locura era demasiado restrictiva y su aplicación podía acarrear importantes problemas legales en nuestro país. Ante ello, se decantaba por la definición más laxa de P. Mata, recogida en el artículo 8 del Código Penal de 1870, por comprender «a todo el que sufre un transtorno mental». El hecho de utilizar la definición del Código Penal, demuestra que los intereses de Esquerdo, estaban más circunscritos a las cuestiones relacionadas con la criminalidad que con la práctica clínica ${ }^{36}$.

En un segundo término, es necesario tener en cuenta factores relacionados con la práctica profesional de los alienistas y con la organización de la asistencia psiquiátrica en la España del último cuarto del siglo pasado.

La ley de Beneficencia de 1849 consagró un doble modelo asistencial: de un lado, una asistencia pública escasamente medicalizada, sumida en el custodialismo, masificada, sin presupuestos y con psiquiatras (cuando los había) de escasa preparación, que se limitó, hasta bien entrado el siglo XX, a ocuparse de unos cuantos miles de locos pobres, sin que produjera obra científica de envergadura ${ }^{37}$. De otro lado, una red asistencial privada, ubicada mayoritariamente en Cataluña y dirigida por médicos, que jugó un papel fundamental en el desarrollo de la psiquiatría como disciplina científica. El objetivo de los alienistas que trabajaban y dirigían estos centros fue reivindicar tanto el carácter científico del alienismo, como el monopolio asistencial de la locura y demostrar la curabilidad de la misma.

Estos alienistas fundamentaban su labor profesional en una mezcla de principios filantrópicos e intereses mercantiles. Así, se mostraban firmes partidarios del tratamiento moral y del non restraint, convencidos de que el aislamiento del enfermo en la institución terapeútica repercutiría en la mejora de su salud. Además, los psiquiatras que ejercían en estos centros defendían el caracter científico y específico del alienismo y reivindicaban ante el Estado y la sociedad el monopolio asistencial de la locura, así como su curabilidad. De hecho, todos ellos con independencia de su concepción de la naturaleza de la locura mostraban un optimismo terapeútico que se

\footnotetext{
35 Ibid, p. 71.

36 ESQUERDO, J.M. a (1882), «Prólogo», en GUISLAIN, J. (1882), Lecciones orales sobre las frenopatías o tratado teórico y práctico de las enfermedades mentales, tomo II, pp. IX-XII.

37 COMELles (1988); HuERTAS (1995b).
} 
reflejaba en sus obras en las que insistían tanto en las virtudes del manicomio como en el alto porcentaje de curaciones ${ }^{38}$.

Sin embargo, estos aspectos humanitarios de su obra respondían a dos objetivos: la legitimación de la profesión, que se movía entre la endeblez y la indiferencia del Estado, y los intereses mercantiles propios de cualquier empresa privada.

El intento por demostrar la curabilidad de la locura y de monopolizar los conocimientos sobre la misma tenía, a pesar de sus indiscutibles fundamentos humanitarios, el doble objetivo de legitimar su labor profesional ante la sociedad y la Administración y de defender los intereses mercantiles de las instituciones que dirigían.

Respecto a este último aspecto, hay que recordar que estas instituciones privadas estaban dirigidas a una clientela acomodada capaz de sufragar los gastos de su estancia, y que buena parte del optimismo psiquiátrico tenía como objetivo la promoción de estos establecimientos entre su potencial clientela. Desde este punto de vista, aunque muchos psiquiatras, como Giné o Esquerdo, fueran somaticistas y proclamaran los orígenes orgánicos de la enfermedad mental, difícilmente podían aceptar la teoría de la degeneración, ligada a la incurabilidad de la enfermedad mental. Admitir lo contrario hubiera sido atacar sus propios intereses científicos y económicos, al poner en peligro las instituciones que dirigían, destinadas a una clientela que buscaban y a la que se ofrecía la curación. ${ }^{39}$

En tercer lugar, el hecho de que los alienistas sean más receptivos al degeneracionismo en sus intervenciones ante los tribunales responde, a nuestro juicio, a los diferentes objetivos que el alienismo persigue en este ámbito y al propio contexto en que se desarrolla el discurso, marcado por la repercusión pública del mismo y del papel del alienista, a todas luces más importante socialmente que el trabajo clínico, demasiado especializado y dirigido a un público minoritario. En cierto modo, la utilización del degeneracionismo ante los tribunales es instrumental.

En este terreno no se trata de mostrar el optimismo ante la curabilidad de la locura y de salvaguardar una clientela acomodada, sino de defender y consolidar la figura del alienista ante otros colectivos profesionales como el resto de los médicos, los juristas y la opinión pública en general.

En este sentido, los viejos esfuerzos del alienismo por demostrar ante los tribunales la enagenación del criminal y su irresponsabilidad jurídica, encuentran en el degeneracionismo un apoyo científico que se manifiesta en la constatación de estigmas físicos y psíquicos como signos de la locura y en la herencia biológica como vehículo de la enfermedad mental. La descripción de los estigmas físicos permite a los alienistas establecer una relación evidente entre la anormal conformación de un individuo y su enfermedad mental y presentarlas ante los juristas y la opinión pública

38 COMELLES (1988); HUERTAS (1995b).

39 COMElles (1988), p. 48; HuERTAS (1995b). 


\section{RICARDO CAMPOS MARÍN}

como la prueba empírica de la enfermedad mental del delincuente. La invisibilidad de los estigmas psíquicos y los obscuros y desconocidos mecanismos de la herencia biológica ${ }^{40}$ posibilita a los alienistas un importante margen de maniobra para reivindicar y presentarse ante la opinión pública como los únicos expertos capacitados para desentrañar los aparentes misterios que se ocultan tras ellos y argumentar científicamente sus explicaciones, a partir precisamente de esa experiencia profesional y técnica que se atribuyen.

Además, todos estos elementos les servirán también para construir una teoría «científica» de la defensa social, y ofrecer a la sociedad la posibilidad de determinar con antelación a la comisión del delito la patología mental y la peligrosidad social del individuo ${ }^{41}$.

Pero además, hay que tener en cuenta también la influencia que ejerció en España la exitosa reinterpretación del degeneracionismo realizada por la escuela positivista italiana. Fueron C. Lombroso y sus discípulos los que en los años 1880 y 1890 catapultaron internacionalmente el degeneracionismo al unirlo a una cuestión que preocupaba a la sociedad finisecular: la criminalidad. En este sentido, en España la repercusión de la obra lombrosiana fue importante durante este período, generando un intenso debate entre sus partidarios y detractores que tuvo una amplia repercusión pública y que fue sin duda una de las principales vías de penetración en nuestro país de las ideas degeneracionistas. Por extensión, esta polémica tuvo también transcendencia en el dominio clínico, aumentando el interés por el degeneracionismo entre los alienistas españoles. Así parece demostrarlo la mencionada obra de V. Garrido, que en su intento por demostrar el papel de experto del alienista en los tribunales, analizaba diversos aspectos de la clínica degeneracionista. También la conferencia Concepto de la degeneración y responsabilidad legal de sus productos mentales, dictada en 1895 por L. Dolsa i Ramón, director del Instituto Frenopático, parece obedecer a esta influencia. Si bien el objetivo de su trabajo era demostrar la existencia en muchos casos de la responsabilidad total o atenuada de los degenerados, la

40 Sobre los motivos del desconocimiento de las leyes de Mendel por parte de los médicos y psiquiatras puede verse: ALBERTI, L (1957). «La medicina experimental y el naturalismo literario». Archivo Iberoamericano de Historia de la Medicina y Antropología médica, 9, pp. 3-66, p. 47; GARCIA MARTINEZ, J. (1984) Aportaciones a la historia de la genética española (1920-1936). Tesina de licenciatura. Facultad de Biología de la Universidad Complutense de Madrid. p. 74. (Inédita)

41 Sobre la capacitación profesional ante los tribunales y la defensa social véanse: MARISTANY, L. (1973), El gabinete del doctor Lombroso.(Delincuencia y fin de siglo en España), Barcelona, Anagrama; AlVAREZ-URÍA (1983); PESET, J.L. (1983), Ciencia y marginación. Sobre negros, locos y criminales, Barcelona, Crítica; TRINIDAD FERNANDEZ, P. (1991), La defensa de la sociedad. Cárcel y delincuencia en España (siglos XVIII-XX), Madrid, Alianza Editorial; GALERA (1991); HUERTAS y MARTíNEZ PÉREZ (1993); MARTÍNEZ PÉREZ, J. (1995b), «Locura y medicina legal: una relación clave para la temprana institucionalización de la psiquiatría en España», en I Congreso de la Sociedad de Historia y Filosofía de la Psiquiatría. Un siglo de Psiquiatría en España, Madrid, Extra Editorial, pp. 69-85. 
primera parte de la conferencia estaba dedicada exclusivamente a los aspectos clínicos de la degeneración, sin relación con la criminalidad, constituyendo además una de las aportaciones españolas más interesantes sobre la clínica degeneracionista ${ }^{42}$.

\section{EL CAMBIO DE ACTITUD DE PRINCIPIOS DE SIGLO.}

En torno a 1900, la psiquiatría española adopta una posición más homogenea respecto a la teoría de la degeneración. Desde la segunda mitad de la década de 1890 , el degeneracionismo comienza a ser aceptado también en el terreno clínico como teoría explicativa de la enfermedad mental, alcanzando en las primeras décadas del presente siglo una posición preeminente entre los psiquiatras, que lo utilizarán normalmente en sus estudios sobre la enfermedad mental. Aunque los problemas de institucionalización de la psiquiatría seguían siendo parecidos a los del último tercio del siglo XIX, y los psiquiatras continuaban en su lucha por demostrar que eran los detentadores de un monopolio científico, surgen una serie de elementos nuevos que van a incidir en su cambio de actitud. En la década de 1890, el degeneracionismo alcanza su mayor nivel de popularización en toda Europa. En 1895, V. Magnan y P.M. Legrain publican su obra Les Dégénérés en la que reformulan desde una perspectiva más acorde con la mentalidad científica del momento, la teoría de Morel ${ }^{43}$. La introducción de elementos evolucionistas, y la radicalización de los postulados somaticistas de la teoría, que equipara la degeneración con la herencia, facilitaron en la coyuntura de fin de siglo una mayor aceptación en los medios científicos de dicha teoría ${ }^{44}$. Practicamente todos los psiquiatras europeos de cierto renombre fueron degeneracionistas durante este período. Pero la expansión del degeneracionismo no se limitó exclusivamente al terreno científico sino que se extendió a otros terrenos como la política, la sociología o la literatura como forma de expresar «científicamente» la percepción de una crisis cultural, histórica y biológica ${ }^{45}$. La célebre saga de los Rou-

42 DOLSA y RAMÓN, L. (1895), Concepto de la degeneración y responsabilidad legal de sus productos mentales. Discurso inagural del año académico de 1895-96 leido en la Academia y Laboratorio de Ciencias Médicas de Cataluña, Barcelona, Imprenta de Henrich y $\mathrm{C}^{\mathrm{a}}$.

43 Magnan, V. y Legrain, P.M. (1895), Les dégénérés. Etat mental et syndromes épisodiques, París, Rueff.

44 GENIL PERRIN, G.P.H. (1913), Histoire des origines et de l'evolution de l'idée de dégénérescence en médecine mentale, París, Alfred Leclerc, Editeur; HUERTAS GARCIA-ALEJO, R. (1987), Locura y Degeneración. Psiquiatría y sociedad en el positivismo francés, Madrid, CSIC; HOCHMANN, J. (1992), «La théorie de la dégénérescence de B.-A. Morel, ses origines et son évolution», en Tort, P. (ed), Darwinisme et Société, París, PUF, pp. 401-412.

45 NYE, R.A. (1985), «Sociolgy and Degeneration: the Irony of Progrees», en CHAMBERLAIN, J.E, GILMAN, S.L. (Eds), Degeneration. The Dark Side of Progress, Nueva York, pp. 49-71; PICK, D. (1989), Faces of Degeneration. A European disorder, c. 1848-c.1918, Cambridge, Cambridge University Press. 
geon-Macquart de Zola, las críticas literarias a la estética decadentista y modernista, la obra de M. Nordau, el impulso de las teorías criminológicas de C. Lombroso, los análisis de los problemas de la clase obrera y sus insurrecciones en clave degeneracionista son buenos ejemplos de esta actitud.

España no permaneció ajena a este impulso del degeneracionismo a finales de si$\mathrm{glo}^{46}$. En lo que se refiere al campo psiquiátrico es indudable que el impulso científico del degeneracionismo en Europa repercutió notablemente en nuestro país. Sin embargo, junto a este elemento existen otros, que tenían su origen en el desarrollo y contingencias de la disciplina psiquiátrica, que merecen ser apuntados para comprender la aceptación del degeneracionismo por el alienismo español.

En primer lugar, hay que destacar que desde la década de 1880 algunos de los manicomios privados de Cataluña, como consecuencia de la inhibición del Estado en sus deberes asistenciales y de su propia lógica mercantil, cayeron en el custodialismo. El caso más significativo fue el del manicomio de San Baudilio de Llobregat, cuyo director y propietario, A. Pujadas, para cubrir la enorme oferta de plazas de su institución, negoció con las Diputaciones Provinciales la provisión de una serie de plazas para enfermos indigentes. Las consecuencias de tal política fue la deserción paulatina de pensionistas, su sustitución con más enfermos pobres, la caída de la institución en el custodialismo y la cronicidad de los enfermos mentales. Entre 18851895, A. Galcerán i Granés se hizo cargo de la dirección de San Baudilio. Aunque en diversos escritos Galcerán sale en defensa de la institución que dirige, la situación real del manicomio parece que continuó degradándose ${ }^{47}$. Si bien es díficil establecer una relación lineal de causa-efecto, lo cierto es que el alienista catalán desde principios de siglo presta mayor atención al degeneracionismo. Sin duda que un psiquiatra somaticista a ultranza como Galcerán, no podía permanecer ajeno al impulso que el degeneracionismo tomó a fines del XIX, pero también es muy posible que su experiencia al frente de San Baudilio, en un momento en que ya no se buscaba ofrecer una mercancía a la burguesía sino gestionar un manicomio de indigentes, pudiera favorecer su paulatino interés por dicha teoría psiquiátrica.

Prueba de su cambio de actitud a partir de 1900, es su prólogo laudatorio a la obra de J.M. ${ }^{a}$ Martínez Valverde, Guía del Diagnóstico de las enfermedades mentales, en la que los postulados degeneracionistas están perfectamente integrados en el conjunto

46 Sobre el ambiente cultural y literario favorable al degeneracionismo en la España del cambio de siglo puede verse: LITVAK L. (1990), «La idea de la decadencia en la crítica antimodernista en España (1888-1910)» y «Temática de la decadencia en la literatura española de fnes del siglo XIX: 1880-1913», en LITVAK, L, España 1900. Modernismo, Anarquismo y fin de siglo, Barcelona, Anthropos, pp. 111127 y 245-25; MARISTANY (1973); MARISTANY, L. (1985) El artista y sus congéneres. Diagnósticos sobre el fin de siglo en España, Barcelona, Tesis doctoral. Inédita; BOIX MARTÍNEZ, R. (1994), Enfermedad y sociedad en la obra de B. Pérez Galdós, Tesis Doctoral. Universidad Autónoma de Madrid. Facultad de Medicina.(Inédita)

47 COMELles (1988), pp. 67-101. 


\section{LA TEORÍA DE LA DEGENERACÓN Y LA PROFESIONALIZACIÓN DE LA PSIQUIATRÍA}

del texto ${ }^{48}$. También en 1900, en un artículo publicado en la Gaceta Médica Catalana sobre el diagnóstico de la locura, Galcerán dedica un epígrafe a la degeneración y en un folleto aparecido en 1908 sostiene que entre las psicopatías ingénitas se encuentran las atípicas, las neurósicas, las degenerativas y las agenésicas. Si bien especifica la existencia de locuras degenerativas, cuyas formas clínicas recuerdan al delirio crónico sistematizado de Magnan, las otras tres psicopatías las considera grados en la escala de la degeneración psíquica ${ }^{49}$. En 1910, con motivo de la fundación de la Sociedad de Psiquiatría y Neurología de Barcelona, nuestro psiquiatra en su discurso inaugural manifestaba la clara vocación higienista de la disciplina y reflexionaba sobre las patologías sociales a partir de la analogía entre el cuerpo humano y la organización de la sociedad, señalando la necesidad de combatir las enfermedades y vicios sociales, entre los que incluía desde «delirios revolucionarios y las guerras religiosas» hasta el pauperismo, la vagancia o la prostitución ${ }^{50}$. El discurso de Galcerán suponía un cambio de primer orden en su trayectoria muy centrada en la clínica y escasamente interesada por los problemas sociales. El salto cualitativo que estaba proponiendo y que tendría repercusiones inmediatas en la política asistencial de la locura diseñada por la Mancomunidad de Cataluña nacía, entre otros factores, de la constatación de que el manicomio como institución terapeútica había fracasado, al convertirse en un mero depósito de enfermos mentales. El salto de la psiquiatría a la sociedad que propugnaba, entroncaba en cierto modo con los planes higienistas de los primeros degeneracionistas franceses ${ }^{51}$.

Un año más tarde, en la Gaceta Médica Catalana, publicó cuatro conferencias sobre «Casuística y terapéutica de la delincuencia», en las que el tono distante del clínico desaparece para tomar claramente partido por el degeneracionismo. En ellas, integra las enseñanzas de Lombroso y su escuela y apunta entre las causas orgánicas de la delincuencia las degeneraciones psicoorgánicas que divide en atavismo, atipia y agenesia. A su juicio, el atavismo se caracteriza «por el predominio de la vida biológica sobre la vida psíquica», y el individuo que la padecía recordaba la anatomía y fisiología del hombre primitivo y de las razas inferiores. Los criminales de este tipo carecían de volición aunque no de razón. Los delincuentes atípicos se distinguían por «la anormalidad de la vida moral», por un «perenne descarrilamiento del psiquismo», en definitiva, por su degeneración psíquica. Por su parte, la degeneración agenésica

\footnotetext{
48 MARTINEZ VAlVERDE, J. (1900), Guía del diagnóstico de las enfermedades mentales con nociones sobre la terapeútica, deontología y medicina legal frenopáticas, Barcelona, José Espasa Editor.

49 GALCERAN I GRANÉS, A. (1900), «Consideraciones sobre la clasificación de las enfermedades mentales a propósito de la adoptada para el servicio clínico del Manicomio de Reus», Gaceta Médica Catalana, XXIII, pp. 521-525 Y 556-561; GALCERÁN I GRANÉS, A. (1908), Diagnóstico Frenopático, Gerona, Imprenta y Librería de Dolores Torres, pp. 15-17.

50 GALCERAN I GRANES (1911), pp. 86-87.

51 HUERTAS (1992), pp. 54 y ss.
} 
se caracterizaba «por el incompleto desarrollo y, a veces, por la falta completa de alguna o de todas las manifestaciones del psiquismo humano». Su principal carencia era la del sentido moral y sus tipos mas corrientes eran los pobres de espíritu, los atrasados, los imbéciles y los idiotas ${ }^{52}$. Por otra parte, dedicaba un epígrafe a la terapéutica social de la degeneración en la que proponía todo un nuevo sistema de corrección y reinserción social del degenerado, basada en el estudio y terapeútica de la naturaleza del individuo y no del tipo de crimen cometido, mostrándose próximo a lo defendido por la escuela positivista italiana ${ }^{53}$. Puede verse por tanto, como Galcerán al abordar un problema propio de la psiquiatría forense no duda en argumentar sus razonamientos desde el degeneracionismo.

Este creciente interés de Galcerán por la degeneración se produjo también en otros psiquiatras e instituciones. La Revista Frenopática Española (1903-1915), órgano científico de los manicomios de San Baudilio de Llobregat, Ciempozuelos, Santa Agueda y Palencia, propiedad de la Orden de San Juan de Dios, es una buena muestra. En sus páginas, asoman numerosos artículos que abordan el degeneracionismo como un todo, sin diferenciar entre la clínica y la psiquiatría forense, e incluso en estadísticas anuales la degeneración aparece como una patología más.

Otros ejemplos del interés que suscitó el degeneracionismo en este período fueron las obras de V. Ots y Esquerdo, formado por J.M. ${ }^{a}$ Esquerdo en el Manicomio de Carabanchel, quien en 1897, publica un folleto, Neurosis y degeneración $n^{54}$, en el que desde un punto de vista estrictamente clínico relaciona ambos fenómenos, o la de $\mathbf{M}$. Bonafonte, director del Manicomio de Zaragoza, que en 1900 publica su tesis doctoral Degeneración y Locura en la que se muestra abiertamente degeneracionista ${ }^{55}$.

Pero junto a la conciencia de que el manicomio ha fracasado en su misión terapeútica y al ambiente científico europeo, hay que tomar en cuenta la incidencia de los acontecimientos de 1898 y del discurso regeneracionista. El regeneracionismo no puede entenderse, como ha señalado Alvarez Uría, sin su correlato de degeneración. 1898 supuso, en este sentido, como 1870 para el caso francés, una toma de conciencia sobre la decadencia del pais ${ }^{56}$. Decadencia, que en el lenguaje científico va a encarnar el término degeneración como constatación de la pérdida de vigor «racial» que padecía el pueblo español. Esta explicación biológica de los problemas de España, que considerará a la herencia morbosa como vehículo, y a la proliferación de

\footnotetext{
52 GalCERAn y Granés, A. (1911), «Casuística y terapeútica de la delincuencia», Gaceta Médica Catalana, pp. 323-330, 323-326.

53 Ibid, pp. 402-406.

54 OTS y ESQUERDO, V. (1897), Neurosis y Degeneración, Madrid, Administración de la Revista de Medicina y Cirugía Prácticas.

55 BONAFONTE NoguÉs, M. (1900), Degeneración y Locura, Zaragoza, Tipografía de Manuel Ventura.

56 Alvarez-Uria, F. (1983), p. 184.
} 
enfermos mentales, criminales, anormales etc., como su principal manifestación, tendrá consecuencias en el discurso psiquiátrico. Máxime cuando los médicos sociales e higienistas se mostrarán decidamente partidarios del degeneracionismo para explicar los problemas del país en clave sociobiológica, convirtiéndose así en actores principales de la introducción de dicha teoría en España ${ }^{57}$. Ejemplo de esta influencia serán las obras de J. Sanchís Banús, G. Rodríguez Lafora, y J. Salas Vaca que abordarán el problema de la degeneración desde postulados médico sociales e incidirán en la explicación sociobiológica de la decadencia de España ${ }^{58}$.

57 CAMPOS MARÍN (1998)

58 SANCHIS BANUS, J. (1916), Estudio médico-social del niño golfo, Madrid, Tip. Excelsior; LAFORA, G.R. (1917), Los niños mentalmente anormales, Madrid, La Lectura; SALAS VACA, J. (1920), Los degenerados en sociedad, Madrid. 